\title{
A search for intergalactic HI gas in the NGC 1808 group of galaxies
}

\author{
M. Dahlem ${ }^{1, \star}$, M. Ehle ${ }^{2,3}$, and S. D. Ryder ${ }^{4}$ \\ 1 Sterrewacht Leiden, Postbus 9513, 2300 RA Leiden, The Netherlands \\ 2 XMM-Newton Science Operations Centre, Apartado 50727, 28080 Madrid, Spain \\ 3 Astrophysics Division, Space Science Department of ESA, ESTEC, \\ 2200 AG Noordwijk, The Netherlands \\ 4 Anglo-Australian Observatory, PO Box 296, Epping, NSW 1710, Australia
}

Received 21 March 2001 / Accepted 27 April 2001

\begin{abstract}
A mosaic of six H I line observations with the Australia Telescope Compact Array is used to search for intergalactic gas in the NGC 1808 group of galaxies. Within the field of view of about $1.4 \times 1.2$ no emission from intergalactic H I gas is detected, either in the form of tidal plumes or tails, intergalactic H I clouds, or as gas associated with tidal dwarf galaxies, with a $5 \sigma$ limiting sensitivity of about $3 \times 10^{18} \mathrm{~cm}^{-2}$ (or $1.4 \times 10^{7} M_{\odot}$ at a distance of $10.9 \mathrm{Mpc}$, for the given beam size of $\left.127^{\prime \prime} .9 \times 77^{\prime \prime} .3\right)$. The H I data of NGC 1792 and NGC 1808 , with a velocity resolution of $6.6 \mathrm{~km} \mathrm{~s}^{-1}$, confirm the results of earlier VLA observations. Simultaneous wide-band $1.34 \mathrm{GHz}$ continuum observations also corroborate the results of earlier studies. However, the continuum flux of NGC 1808 measured by us is almost $20 \%$ higher than reported previously. No radio continuum emission was detected from the type Ia supernova SN1993af in the north-eastern spiral arm of NGC1808. A comparison of NGC 1792 and NGC 1808 shows that it is not primarily the total energy input that makes the big difference between the starburst-related outflow in NGC 1808 and the absence of such extraplanar features in NGC 1792, but the area over which the energy released by stellar winds and supernovae is injected into the ISM.
\end{abstract}

Key words. galaxies: individual: NGC 1792, NGC 1808 - galaxies: general - galaxies: ISM - galaxies: interactions - intergalactic medium

\section{Introduction}

It has been argued by us earlier (Dahlem et al. 1990, 1994; Dahlem 1992, hereafter D92) that the starburst activity in the Sérsic-Pastoriza galaxy NGC 1808 (Sérsic \& Pastoriza 1965) and the high level of star formation (SF) in its nearby companion galaxy NGC 1792 might have been triggered by a gravitational interaction between these galaxies. This scenario had originally been proposed by van den Bergh (1978). Indirect evidence comes, e.g., from a warp of the stellar and gaseous disk of NGC 1808 and a pronounced asymmetry in the spatial distribution of H I gas and H II regions in NGC 1792. Except for the warp indicated above, which includes the stellar population in the outer " $\theta$ " -shaped spiral arms of NGC 1808 (Koribalski et al. 1993; hereafter K93), the stellar distributions in both systems appear to be only slightly disturbed, arguing in favour of a distant passage. In particular the HI gas in

Send offprint requests to: M. Dahlem,

e-mail: mdahlem@eso.org

* Present address: Alonso de Cordova 3107, Vitacura, Casilla 19001, Santiago 19, Chile.
NGC 1792 shows another peculiarity that made us speculate about the possibility of gas stripping in its outermost parts, namely a very notable sharp drop of the measured H I surface brightness (and thus, under the assumption of optically thin emission, line of sight column density) within the optically visible stellar disk (D92). This is unusual and normally found only in interacting systems, like e.g. galaxies in the Virgo cluster (Kenney \& Young 1989). One galaxy detected by us exhibiting such behaviour without a nearby interaction partner is NGC 3175 (Dahlem et al. 2001). Most spiral galaxies, on the other hand, have detectable H I gas far beyond their optically visible stellar disks (e.g. Bosma 1981).

In a number of groups of galaxies intergalactic H I has been found in the form of tails and/or plumes, as for example in the NGC 4631 group (Weliachew et al. 1978) and the Leo triplet, which includes NGC 3628 (Haynes et al. 1979). Such detections are important, because tidal features can provide strong constraints on $n$-body simulations of the dynamics of pairs of interacting galaxies (see e.g. Combes 1978 for a model describing the data by Weliachew et al. 1978). Such models help tremendously in establishing 
interaction timescales and understanding streaming motions of gas and stars in the individual systems, often providing the only way to explain the properties of the observed galaxies. NGC 1792 and NGC 1808 belong to a small group of galaxies (Garcia 1993), for which we adopt here, as done by us previously, the distance of NGC 1808 of $D=10.9 \mathrm{Mpc}$ (based on $H_{0}=75 \mathrm{~km} \mathrm{~s}^{-1} \mathrm{Mpc}^{-1}$ and a virgocentric infall velocity of $300 \mathrm{~km} \mathrm{~s}^{-1}$ ). The disturbances mentioned above suggest a tidal interaction (e.g., Dahlem et al. 1994). However, the evidence for an interaction in the past is circumstantial, which prompted us to search for intergalactic H I gas in this group so as to obtain direct proof of tidal forces at work. To that end we used the very compact 375-m array of the Australia Telescope Compact Array (ATCA; Frater et al. 1992) ${ }^{1}$ to search for intergalactic H I gas in the NGC 1808 group, conducting observations centred between NGC 1792 and NGC 1808, the two dominant group members. With a shortest baseline of $31 \mathrm{~m}$ and good uv coverage on baselines up to $459 \mathrm{~m}$ (discarding data from antenna 6, which is located at a distance of $3 \mathrm{~km}$ from the five moveable antennae), this array, being more compact than the VLA D array, is very wellsuited to conduct such a search for extended low surface brightness emission.

\section{Observations and data reduction}

Our observations were carried out in late 1996; the most important dates and parameters are listed in Table 1. Of the 12:45 hours about 1:45 $\mathrm{h}$ were spent on flux, bandpass and phase calibration. The rest of the time was split evenly among six pointing directions. One was centred on NGC 1792, one on NGC 1808 and the remaining four between the two galaxies, as specified in the table.

All data were calibrated in the usual way, using the MIRIAD package installed at the Australia Telescope National Facility. We assumed a continuum flux density at $1.42 \mathrm{GHz}$ of PKS B1934-638 of 15.01 Jy. PKS B0537441, which served as phase calibrator, was observed at regular intervals during the observations. The data for the six pointings were separated from each other in the uv plane of the visibilities and calibrated separately. Subsequently the data were recombined using the MIRIAD task UVAVER and then jointly cleaned, using the task MOSSDI, and restored with a common angular resolution (Full Width at Half Maximum) of $F W H M=$ 127 !' $9 \times 77^{\prime \prime} .3$. For our attempt to detect extended low surface brightness emission, two original line channels each were averaged, resulting in a velocity resolution of $6.6 \mathrm{~km} \mathrm{~s}^{-1}$. The resulting data cube has 160 channels of $6.6 \mathrm{~km} \mathrm{~s}^{-1}$ width each and covers a field of view (FOV) of approximately $1.4 \times 1.2$. The major axis of this elongated FOV is oriented along the line connecting NGC 1792

\footnotetext{
1 The Australia Telescope is funded by the Commonwealth of Australia for operation as a National Facility managed by CSIRO.
}

Table 1. Details of ATCA 375-m array observations.

\begin{tabular}{lc}
\hline Observing Date & 1996, Dec. 8-9 \\
Net Observing Time & $11: 00 \mathrm{~h}$ \\
Shortest Baseline & $31 \mathrm{~m}$ \\
Longest Baseline $^{\mathrm{a}}$ & $459 \mathrm{~m}$ \\
Flux Calibrator & $1934-638$ \\
Phase Calibrator & $0537-441$ \\
Primary Beam $(F W H M)$ & $33^{\prime}$ \\
Restored Beam $(F W H M)$ & $127^{\prime \prime} 9 \times 77^{\prime \prime} 3$ \\
Bandwidth (helio. vel. $)$ & $620-1675 \mathrm{~km} \mathrm{~s}^{-1}$ \\
Velocity Resolution & $6.6 \mathrm{~km} \mathrm{~s}^{-1}$ \\
Sensitivity per Channel & $5 \mathrm{mJy} \mathrm{beam}{ }^{\mathrm{b}}$ \\
$S_{\mathrm{HI}} / T_{\mathrm{B}}$ Conversion Factor & $62.2 \mathrm{Jy} / \mathrm{K}$ \\
Pointing $1(\alpha, \delta(2000))$ & $05: 05: 13.8,-37: 58: 49$ \\
Pointing $2(\alpha, \delta(2000))$ & $05: 05: 40.9,-37: 35: 00$ \\
Pointing $3(\alpha, \delta(2000))$ & $05: 06: 52.8,-37: 40: 06$ \\
Pointing $4(\alpha, \delta(2000))$ & $05: 06: 52.9,-37: 49: 28$ \\
Pointing $5(\alpha, \delta(2000))$ & $05: 07: 15.6,-37: 54: 33$ \\
Pointing $6(\alpha, \delta(2000))$ & $05: 07: 42.1,-37: 30: 44$ \\
\hline
\end{tabular}

Notes to Table 1:

a) Discarding data from antenna 6 .

b) $1-\sigma \mathrm{rms}$ at the centre of the field of view.

c) Conversion from antenna brightness temperature to flux density units for the angular resolution of our data of $127 . .9 \times$ 77.'3 (ATCA Users Guide; October 1999).

and NGC 1808. The data were also corrected for the effect of primary beam attenuation across the FOV.

Continuum subtraction was performed by subtracting the average of the line-free channels from each of the channels containing line emission in each of the sub-datasets.

\section{Results and discussion}

\subsection{HI in NGC 1792 and NGC 1808}

Our map of the integral H I line emission from NGC 1792, NGC 1808 and the intergalactic space in between is displayed in Fig. 1. This map was created using the AIPS routine MOMNT to calculate three "moment maps" (total intensity, velocity field and velocity dispersion) from the original data $c u b e^{2}$, before applying the primary beam

\footnotetext{
2 Note that the "standard" procedure for the production of "moment maps" is to smooth the original data cube and use a user-defined flux-density threshold in that smoothed version as a "mask" to circumscribe features that are considered to be real emission, while data points below the threshold are blanked. In this process, the signal-to-background ratio of the moment maps is increased significantly. However, since features below a certain threshold are removed systematically, there is no measure any more of the inherent thermal noise of the data. Such blanked moment maps will be shown below. Here, in the display of the total FOV, we want to present an image including the true noise of the data, especially in the region of particular interest, between NGC 1792 and NGC 1808.
} 


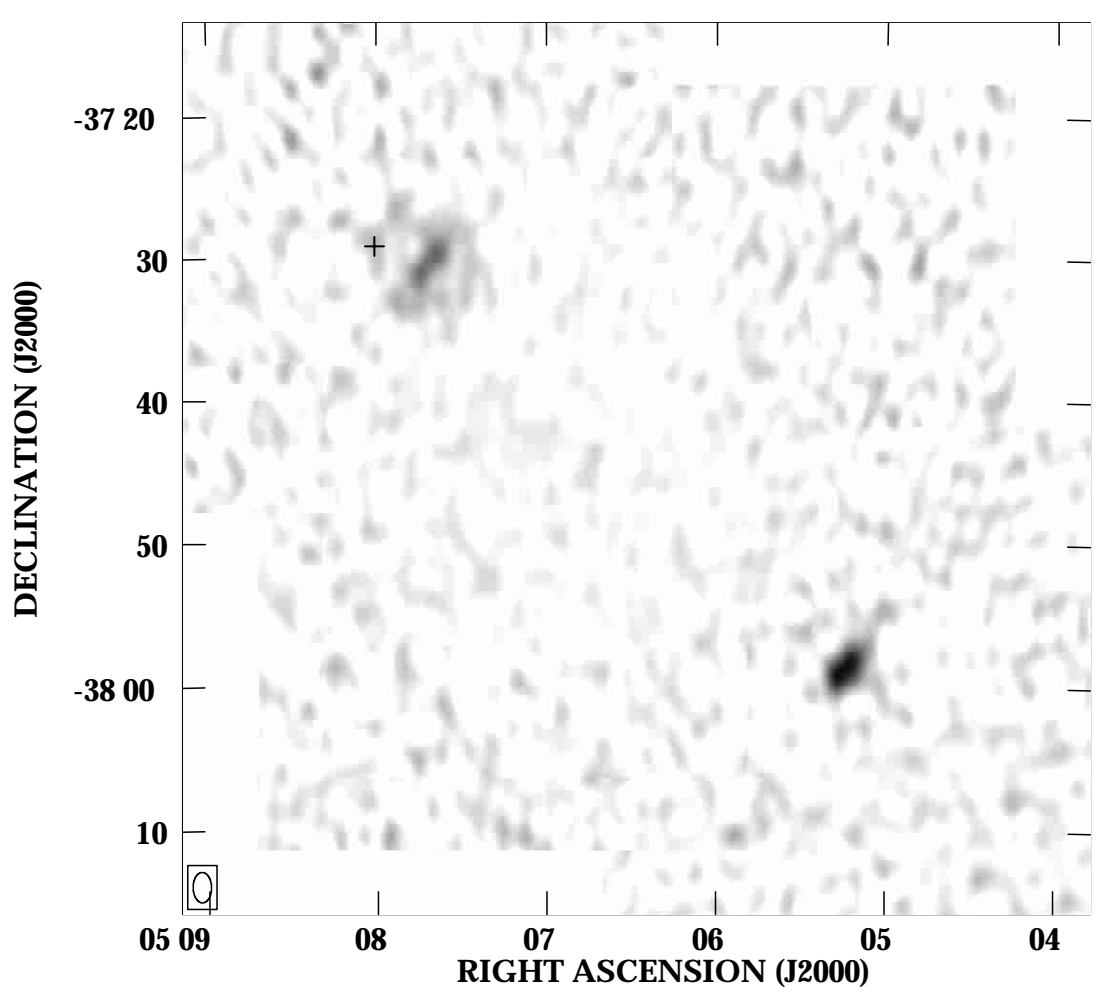

Fig. 1. Image of the total H I line emission of NGC 1808 (upper left) and NGC 1792 (lower right), the two dominant members of the NGC 1808 group of galaxies, and their surroundings. The cross in the northeastern spiral arm of NGC 1808 marks the position of SN1993af. The angular resolution of the data is indicated in the lower left; the linear grey scale ranges from 0 to the maximum observed surface brightness of $1.912 \mathrm{Jy}_{\text {beam }}{ }^{-1}$.

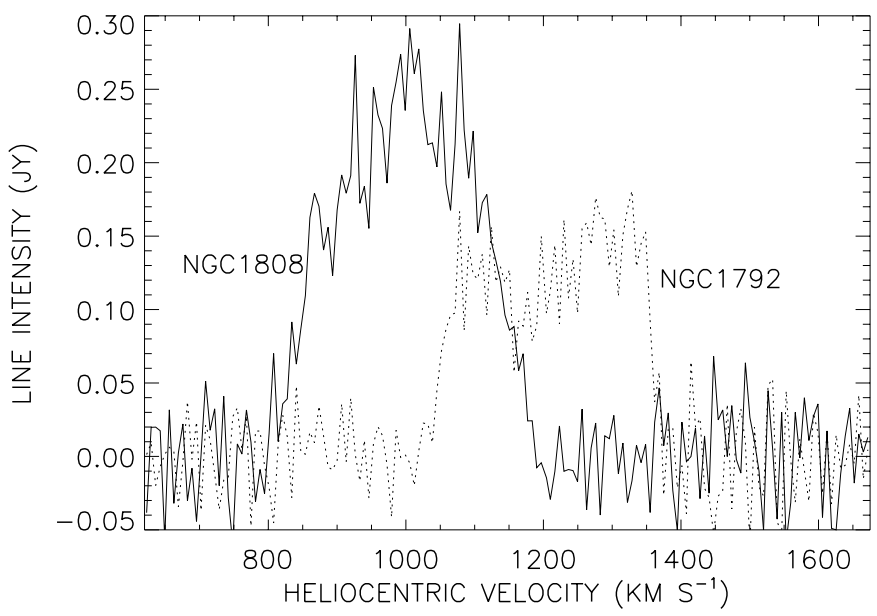

Fig. 2. Integral H I line spectra of NGC 1792 (dotted line) and NGC 1808 (solid line) superimposed onto each other to illustrate the overlap in velocity space and the relative intensities.

correction. It shows clearly resolved HI emission from both galaxies. The 1- $\sigma$ rms noise of the data at the positions of both galaxies is of order $10 \mathrm{mJy} \mathrm{beam}^{-1}$ per channel; at the centre of the FOV, where all six pointings overlap within the $F W H M$ of the primary beam, it is approximately $5 \mathrm{mJy}$ beam $^{-1}$ per channel.

Assuming optically thin $\mathrm{H}$ I line emission $(\tau \ll 1)$, the observed flux densities can be converted into H I column
Table 2. Integral properties of NGC 1792 and NGC 1808.

\begin{tabular}{lrr}
\hline Property & NGC 1792 & NGC 1808 \\
\hline$W_{20}\left(\mathrm{~km} \mathrm{~s}^{-1}\right)$ & $326 \pm 10$ & $342 \pm 10$ \\
$W_{50}\left(\mathrm{~km} \mathrm{~s}^{-1}\right)$ & $295 \pm 10$ & $266 \pm 10$ \\
$v_{\text {hel }}\left(\mathrm{km} \mathrm{s}^{-1}\right)$ & $1207 \pm 10$ & $1001 \pm 10$ \\
$f_{\mathrm{HI}}\left(\mathrm{Jy} \mathrm{km} \mathrm{s}^{-1}\right)$ & $39.5 \pm 5$ & $63.4 \pm 6$ \\
$f_{1.34}(\mathrm{mJy})$ & $325 \pm 10$ & $616 \pm 15$ \\
$\log \left(L_{1.34}\right)\left(\mathrm{W} \mathrm{Hz}^{-1}\right)$ & 21.66 & 21.94 \\
\hline
\end{tabular}

densities following the relation (Eq. (12.2) by Giovanelli \& Haynes 1988)

$N(\mathrm{H})=1.83 \times 10^{18} T_{\mathrm{B}}$,

with the $T_{\mathrm{B}} / S_{\mathrm{HI}}$ conversion factor from Table $1 . N(\mathrm{H})$ is the $\mathrm{H}$ I column density in units $\mathrm{cm}^{-2}$ and $T_{\mathrm{B}}$ the antenna brightness temperature in units K. For our observations a line surface brightness of $1 \mathrm{Jy}^{\text {beam }}{ }^{-1}$ corresponds to a column density of $N(\mathrm{H})=1.14 \times 10^{20} \mathrm{~cm}^{-2}$ (assuming that the size of the observed structure is comparable to the beam size).

We will first introduce some global emission properties of both galaxies, before turning to details. The total H I line spectra of both NGC 1792 and NGC 1808 are displayed in Fig. 2. The resolution of the current data, of 
Table 3. Global properties ${ }^{\mathrm{a}}$ of NGC 1792 and NGC 1808.

\begin{tabular}{lccc}
\hline Property & Symbol & NGC 1792 & NGC 1808 \\
\hline Absolute blue magnitude $^{\mathrm{b}}$ & $M_{\mathrm{B}}(\mathrm{mag})$ & $-20.15 \pm 0.10$ & $-20.24 \pm 0.10$ \\
Total blue luminosity & $L_{\mathrm{B}}\left(L_{\odot}\right)$ & $1.6 \times 10^{10}$ & $1.7 \times 10^{10}$ \\
Total H I gas mass & $M_{\mathrm{HI}}\left(M_{\odot}\right)$ & $1.1 \times 10^{9}$ & $1.8 \times 10^{9} \mathrm{c}$ \\
Total virial mass & $M_{\mathrm{T}}\left(M_{\odot}\right)$ & $0.54 \times 10^{11} \mathrm{~d}$ & $1.1 \times 10^{11 \quad \mathrm{e}}$ \\
H I gas to total mass ratio & $M_{\mathrm{HI}} / M_{\mathrm{T}}$ & 0.021 & $0.016^{\mathrm{c}}$ \\
H I gas to blue light ratio & $\frac{M_{\mathrm{HI}}\left(\frac{M_{\odot}}{L_{\odot}}\right)}{L_{\mathrm{B}}}$ & 0.07 & $0.10^{\mathrm{c}, \mathrm{e}}$ \\
Total mass-to-light ratio & $\frac{M_{\mathrm{T}}}{L_{\mathrm{B}}}\left(\frac{M_{\odot}}{L_{\odot}}\right)$ & 3.4 & 6.5 \\
Optical colour index & $B-V\left(\mathrm{mag}^{\mathrm{b}}\right)$ & $0.68 \pm 0.14$ & $0.82 \pm 0.14$ \\
Total FIR luminosity & $L_{\mathrm{FIR}}\left(L_{\odot}\right)$ & $0.63 \times 10^{10} \mathrm{~d}$ & $2.4 \times 10^{10 \quad \mathrm{f}}$ \\
Supernova Rate & $\nu_{\mathrm{SN}}\left(\mathrm{yr}^{-1}\right)$ & 0.45 & 0.87 \\
Star Formation Rate & $S F R\left(M_{\odot} \mathrm{yr}^{-1}\right)$ & 11.0 & 21.2 \\
Total Normalised $S F R$ & $\frac{S F R}{A_{\mathrm{SF}}}\left(\frac{M_{\odot}}{\mathrm{yr} \mathrm{kpc}^{2}}\right)$ & 0.072 & $34.1^{\mathrm{h}}$ \\
\hline
\end{tabular}

Notes to Table 3:

a) All values without a note to a reference are calculated by us based on $D=10.9 \mathrm{Mpc}$.

b) Both $B$ and $V$ magnitudes are from the RC3; the distance modulus for $D=10.9 \mathrm{Mpc}$ is -30.19 .

c) Lower limit because of intrinsic self-absorption.

d) From Dahlem (1992).

e) From Koribalski et al. (1993).

f) From Dahlem et al. (1990).

g) For stars with $M \geq 5 M_{\odot}$.

h) Central starburst only; see text.

$6.6 \mathrm{~km} \mathrm{~s}^{-1}$, is about 3 times as high as that of earlier observations (D92; K93). From these new spectra we derive the total widths of the integral $\mathrm{HI}$ lines at the $20 \%$ - and $50 \%$-level of the maximum, $W_{20}$ and $W_{50}$, respectively, the heliocentric systemic velocities, $v_{\text {sys }}$, and the total $\mathrm{H}$ I line fluxes, $f_{\mathrm{HI}}$. $f_{\mathrm{HI}}$ is calculated as the sum over all bins with line emission from each galaxy, multiplied by the bin width of $6.6 \mathrm{~km} \mathrm{~s}^{-1}$. These values are listed in Table 2 .

All measurements of $f_{\mathrm{HI}}, W_{20}$ and $v_{\text {sys }}$ confirm, within the error margins, earlier results obtained from VLA observations (D92; K93). A few global properties derived from these global H I data and other data are collated in Table 3.

Kinematic parameters, such as the kinematic position angle, $P A$, and the inclination angle, $i$, and their behaviour as a function of radial distance from the galaxy centre, can be best constrained from the earlier, higher-resolution VLA data.

\subsubsection{NGC 1792}

The HI distribution in NGC 1792 (Fig. 3) is compact. Not only is the extent of the HI disk small compared to the stellar disk (D92), but with values up to about $3 \times 10^{20} \mathrm{~cm}^{-2}$ the $\mathrm{H}$ I column densities are quite high (see Fig. 1). Note that we use here, contrary to Fig. 1, the total intensity map created by routine MOMNT, using a smoothed version of the original datacube as a mask to avoid summing up noise in areas where no line emission is present. This leads to an improved signal-to-background ratio compared to the map in Fig. 1 (but leaves no sensible background "noise"). Even with its lower angular resolution our current map corroborates the steep outer gradient of the H I emission distribution seen in the earlier VLA observations (D92), which is reminiscent of a sharp radial cutoff.

Our data also confirm the regularity of the H I velocity field of NGC 1792 (not displayed).

\subsubsection{NGC 1808}

Our H I map of NGC 1808 (Fig. 4) clearly resolves the inner disk and the outer spiral arms. Note that we use almost the same contour levels in Figs. 3 and 4, with only a few more densely spaced low-level contours to display the outer regions of NGC 1808. The H I distribution is consistent with earlier results (e.g., Saikia et al. 1990; K93). The surface brightness and thus column density of the $\mathrm{HI}$ emission from the inner disk is relatively low (about $0.2-1.0 \times 10^{20} \mathrm{~cm}^{-2}$, typically). That of the outer spiral arms is yet lower, with values below $0.2 \times 10^{20} \mathrm{~cm}^{-2}$, typically. Part of the central H I gas is not visible in emission because of intrinsic self-absorption along the line of sight towards the nuclear region (see K93 and Koribalski et al. 1996 for details). Therefore, the total H I line flux measured is a lower limit (Table 2). The cross marks the position of SN1993af, which will be discussed below.

The velocity field obtained from our data (not displayed) exhibits the same general features as observed in 

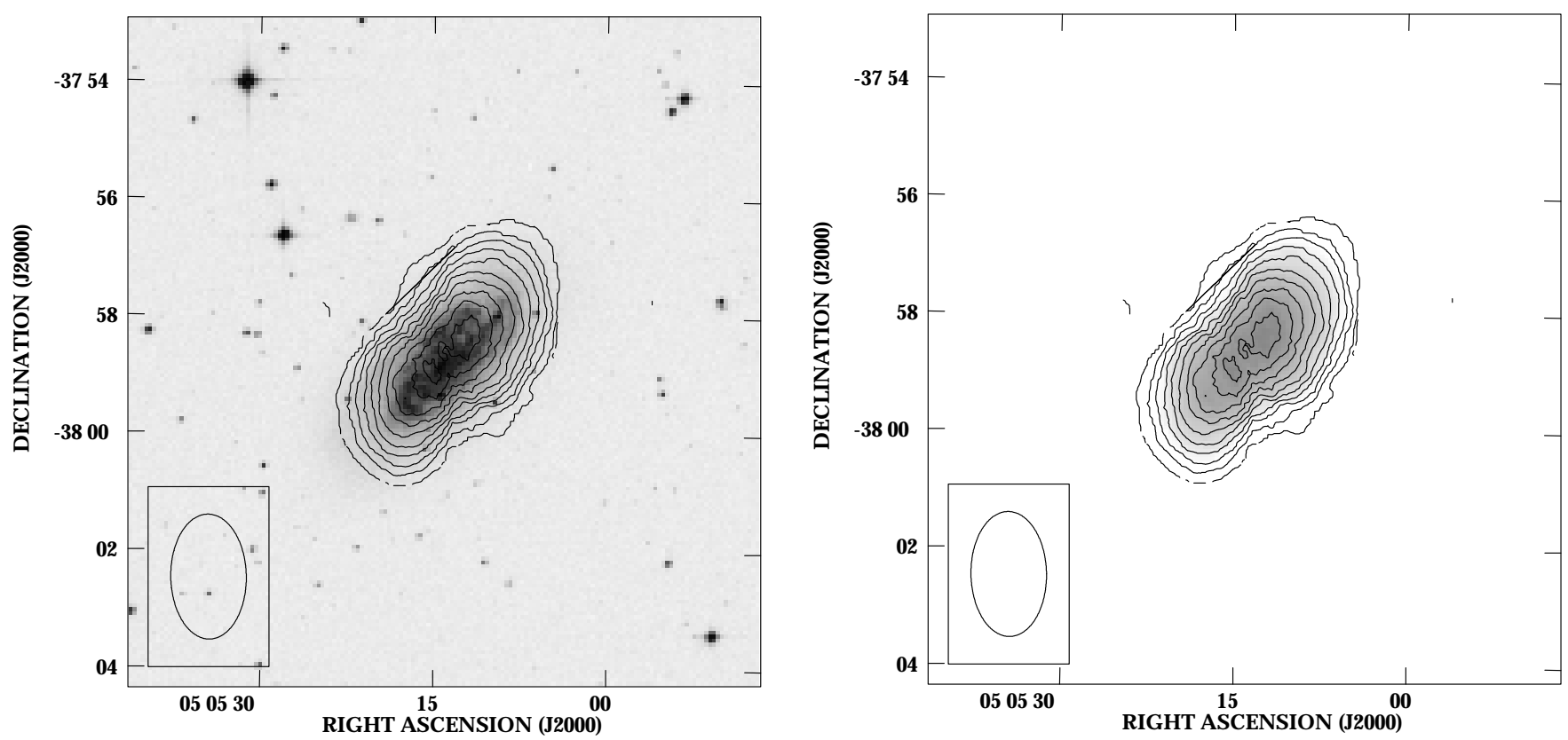

Fig. 3. Contours of the total H I line emission of NGC 1792, overlaid on a DSS optical image (left panel) and contours with underlying grey scale (right panel). The contour levels are $0.027,0.11,0.32,0.54,0.76,0.97,1.19,1.41,1.62,1.84,2.06 \times 10^{20} \mathrm{~cm}^{-2}$; the angular resolution is indicated in the lower left.
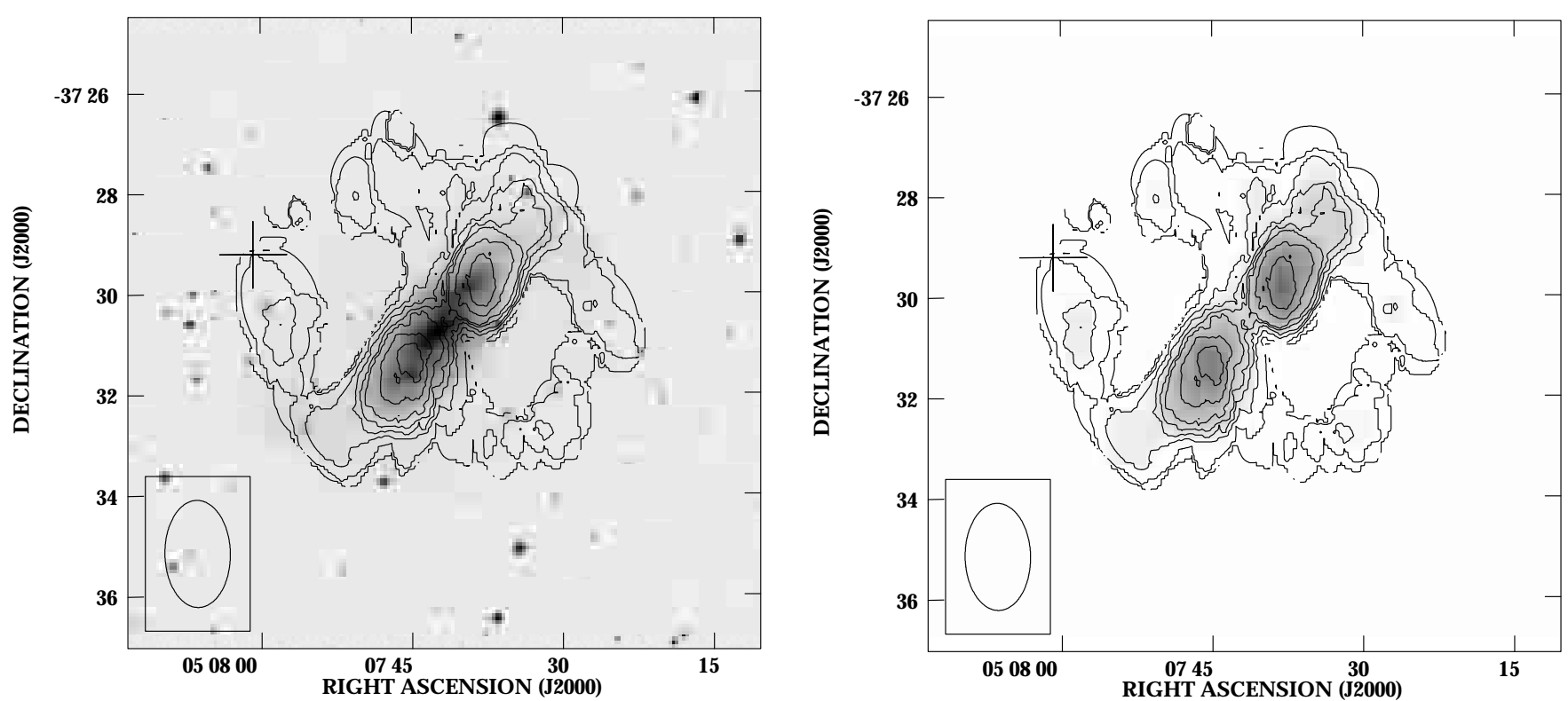

Fig. 4. Contours of the total H I line emission of NGC 1808, overlaid on a DSS optical image (left panel) and contours with underlying grey scale (right panel). The contour levels are $0.027,0.054,0.11,0.22,0.32,0.43,0.54,0.76,0.97 \times 10^{20} \mathrm{~cm}^{-2}$, the angular resolution is indicated in the lower left. The cross again marks the position of SN1993af.

earlier VLA observations (K93), including the skewed appearance due to non-circular gas motions.

\subsubsection{A comparison of NGC 1792 and NGC 1808}

It is directly visible from Fig. 1 that the distribution of $\mathrm{HI}$ in NGC 1792 is much more compact than that of NGC 1808. The extent of the H I distribution in NGC 1792 is smaller than that of its stellar disk and at the same time the surface brightnesses in the disk is also higher. After correction for the different inclination angles, the H I column densities in NGC 1792 are still higher than in NGC 1808, indicating that the mean gas density is higher.

These features of the H I distributions in both galaxies can account for the observed distribution of SF activity: while NGC 1808 has a circumnuclear starburst ring, NGC 1792 exhibits widespread SF over large parts of its disk. The low H I column densities in the $\theta$-shaped outer 
spiral arms of NGC 1808 suggest that there are no signs of SF there because of low gas densities. A comparison of the SF properties of both galaxies will follow below, in the context of our radio continuum images (Sect. 3.3.3).

The rotational behaviour of both galaxies, NGC 1792 and NGC 1808, and the relative alignment of their spin vectors was decribed by us in D92 (Fig. 5).

\subsection{No intergalactic $\mathrm{HI}$ between NGC 1792 and NGC 1808}

Thus, the current observations corroborate earlier results from dedicated studies of HI in both NGC1792 and NGC 1808. But, more importantly, they represent the up to now most sensitive search for intergalactic H I gas in the vicinity of this pair of spirals.

The sensitivity of our data is illustrated by the detection of H I in the outer spiral arms of NGC 1808, despite the fact that the galaxy was observed in only part of the pointings. These spiral arms have relatively low column densities on the order of $10^{19} \mathrm{~cm}^{-2}$. Near the centre of our FOV, the $1-\sigma$ rms noise is $5 \mathrm{mJy}^{\text {beam }^{-1}}$ per channel so that structures with column densities of $\gtrsim 3 \times 10^{18} \mathrm{~cm}^{-2}$ should have been detected at the $5-\sigma$ confidence level. Based on a fiducial extent of roughly $5 \times 5 \mathrm{kpc}$ (one beam size) and a requirement to have a $5-\sigma$ signal in three consecutive channels, this translates into a lowest detectable line flux of $f_{\mathrm{HI}}(\mathrm{min}) \simeq 0.5 \mathrm{Jy} \mathrm{km} \mathrm{s}^{-1}$. Using the relation by Roberts (1975),

$M_{\mathrm{HI}}=2.356 \times 10^{5} D^{2} f_{\mathrm{HI}}\left[M_{\odot}\right]$,

where $D$ is the distance in units $\mathrm{Mpc}$, and assuming optically thin emission, this corresponds to a minimum detectable $\mathrm{H}$ I gas mass of approximately $1.4 \times 10^{7} \mathrm{M}_{\odot}$ (or $5.6 \times 10^{5} M_{\odot} \mathrm{kpc}^{-2}$ ) at the distance of the NGC 1808 group. Near the boundaries of the FOV, where both NGC 1792 and NGC 1808 are located, these values increase to approximately $3 \times 10^{7} M_{\odot}$ (or $1.2 \times$ $10^{6} M_{\odot} \mathrm{kpc}^{-2}$ ). The velocity range covered by our observations is $1055 \mathrm{~km} \mathrm{~s}^{-1}$, from 620 to $1675 \mathrm{~km} \mathrm{~s}^{-1}$ (Table 1), centred at $1148 \mathrm{~km} \mathrm{~s}^{-1}$, i.e. between the systemic velocities of NGC 1792 and NGC 1808. This makes it unlikely that any gas between NGC 1792 and NGC 1808 was missed in redshift space.

We used not only the data with the highest possible angular and velocity resolution, but also various smoothed versions of the data cube. No significant $\mathrm{H}$ I emission was detected that is not displayed in Figs. 1, 3 and 4.

It is evident from Fig. 1 that, despite the use of a very compact interferometer configuration, no $\mathrm{H}$ I gas could be detected in the space between NGC 1792 and NGC 1808 and in their immediate surroundings, at the sensitivity of the data. Only small amounts of diffuse H I gas might have eluded detection, as quantified above. This is not entirely unexpected because, with the gas-to-total mass ratios listed in Table 3, neither of the two galaxies is prominently H I-deficient when compared with the large sample used by Roberts \& Haynes (1994) to determine the "typical" properties of galaxies of different Hubble types.

\subsubsection{Absence of tidal features}

At the boundaries of the HI emission distributions of both galaxies, NGC 1792 and NGC 1808, no plumes, tails or other previously undetected tidal features were found at a significant level in addition to the HI emission features already known from dedicated observations centred on the individual galaxies, with a detection limit of order $3 \times 10^{7} M_{\odot}$.

These results imply that the interaction of the pair NGC 1792 and NGC 1808 that might have caused the observed disturbances (D92; K93) must have left the galaxies with most of their gas. Therefore, a far-field encounter is still the most likely explanation of all observations of these systems.

Our results leave no doubt that the NGC 1808 group does not lend itself to dynamical studies by means of numerical modeling, because there are no distinct features that could sensibly constrain the boundary conditions for $n$-body simulations to model a putative interaction.

\subsubsection{No dwarf galaxies detected}

It is also interesting to note that our observations have not revealed any signs of $\mathrm{H}$ I emission from previously unknown dwarf galaxies near NGC 1792 or NGC 1808, as found in other groups like for example the NGC 4666 group of galaxies (Walter et al., in preparation), see also e.g. Brinks (1990).

This again implies that there are no significant amounts of debris in the form of tidal dwarf galaxies in the NGC 1808 group similar to those found for the first time at the end of the tidal arms of the "Antennae" galaxies (NGC 4038/39, Mirabel et al. 1992) and later e.g. in the Leo Triplet of galaxies (Chromey et al. 1998).

\subsubsection{No primordial intergalactic $\mathrm{HI}$ clouds}

Our results also bear some significance in the context of the hypothesis originally proposed by Oort (1966) and recently refined by Blitz et al. (1999) that Galactic H I highvelocity clouds (HVCs) might be primordial H I gas clouds raining down on the Galaxy. Assuming that similar H I gas clouds exist in external groups of galaxies, these should be detectable with the sensitivity of our observations.

Although our data do not span the entire NGC 1808 group of galaxies, the non-detection of intergalactic $\mathrm{H}$ I at the sensitivity stated above implies a constraint on the possible existence of intergalactic primordial H I clouds. No clouds are detected down to a mass of about $10^{7} M_{\odot}$. This implies that such clouds, if existent, cannot form a significant fraction of the total H I gas mass of that part of the NGC 1808 group of galaxies around the two spirals NGC 1792 and NGC 1808 sampled by our observations. 
This, in turn, constrains the role that such primordial gas can play in forming HVCs raining down on the two galaxies NGC 1792 and NGC 1808, following the reasoning by Zwaan (2001). In the NGC 1792/NGC 1808 system intragroup $\mathrm{H}$ I gas clouds cannot play a significant role as the potential progenitors of HVCs. This implies that our observations cannot lend any support to the theory by Oort (1966) and Blitz et al. (1999). Detailed calculations and simulations based on $\mathrm{H}$ I observations of five groups of galaxies are given by Zwaan (2001) and in the references therein.

\subsubsection{No intergalactic medium at all?}

This non-detection of $\mathrm{HI}$ emission on different spatial scales does not exclude the possibility that an intergalactic medium might exist. However, our data put very tight constraints on the properties of such a medium. If located between NGC 1792 and NGC 1808 and if in the form of $\mathrm{H}$ I, this gas must have very low column densities $\left(\lesssim 3 \times 10^{18} \mathrm{~cm}^{-2}\right)$. There might also be H I gas outside the field of view of our observations, farther away from the two galaxies, towards the other group members (Garcia 1993). Although these are at yet larger projected distances from NGC 1792 and NGC 1808 and/or have lower total masses, it is conceivable that other members of the group, such as e.g. the late-type system ESO 305-G009 at a recession velocity of $1025 \mathrm{~km} \mathrm{~s}^{-1}$, might have taken part in a past interaction.

One might hypothesise whether any intergalactic gas might exist in the form of other phases. For example, at least part of the gas could possibly be too warm to be visible in H I emission. Donahue et al. (1995) detected a diffuse $\mathrm{H} \alpha$-emitting medium around NGC 4631, which might also be related to the softest observed X-ray emission at $k T \simeq 0.05 \mathrm{keV}$ (Dahlem et al. 1998). Searches which could detect warm, $\mathrm{H} \alpha$-emitting gas in the NGC 1808 group of galaxies have not yet been conducted. But it is unlikely that such gas exists, because warm ionised gas should normally be accompanied by a neutral component, as in the case of the NGC 4631 group, which we did not detect around NGC 1792/1808. Both the sensitivity and the field of view of the ROSAT observations of NGC 1792 and NGC 1808 (Dahlem et al. 1994; Junkes et al. 1995) should have led to the detection of a hot $\left(\sim 10^{6} \mathrm{~K}\right)$ intergalactic medium, if such should exist. However, no hot ionised gas was found.

At the other end of the energy scale, the gas might be too cold to be seen in emission. Our H I emission observations trace only warm H I gas. Cold neutral hydrogen gas would not show up in our data, being only observable in absorption against background sources. The gas might be yet colder and exist in the form of molecular gas. However, such gas, outside self-shielding gas clouds and in the absence of significant amounts of shielding dust in galaxy disks, is exposed to the intergalactic UV radia- tion field and thus expected to be heated to temperatures that would make it visible in emission (Shull et al. 1999).

A search for signatures of stellar plumes or tails on deep optical images (see Chromey et al. 1998 for an example) in member galaxies of the NGC 1808 group of galaxies, including ESO 305-G009, also led to a negative result (D. Malin; private communication).

Although being far from conclusive, all these arguments make it very unlikely that there are significant amounts of intergalactic gas in the NGC 1808 group of galaxies, at least in the form of gas phases that are presently known to us (and observable).

\section{3. $1.34 \mathrm{GHz}$ continuum imagery}

The setup of the ATCA allows for parallel H I observations at one intermediate frequency (IF) and continuum observations at the second IF. Therefore, $1.34 \mathrm{GHz}$ radio continuum images with a bandwidth of $84 \mathrm{MHz}$ were obtained simultaneously, which we can compare with the existing data in the literature (cf. Table 2). The map of the same field of view as the H I total intensity image (Fig. 1) is displayed in Fig. 5. NGC 1792 and NGC 1808 are the most prominent radio continuum sources in the field of view. In the lower left an arc of an imperfectly cleaned beam fringe of NGC 1808 is visible. The rms noise of the map is about $1 \mathrm{mJy}^{\text {beam }}{ }^{-1}$ in the central region and about 2 mJy beam $^{-1}$ further out, near NGC 1792 and NGC 1808.

\subsubsection{NGC 1792}

The $1.34 \mathrm{GHz}$ continuum emission distribution of NGC 1792 derived from the present data is consistent with our earlier map (D92). The total flux measured from the present data is $325 \pm 10 \mathrm{mJy}$. This is compatible with our earlier measurement of $324 \pm 20 \mathrm{mJy}$ at $1.41 \mathrm{GHz}$ (D92), which implies that the flux calibration of our observations is consistent with the earlier VLA observations.

The radio continuum surface brightness is high, which is consistent with the high H I surface brightnesses (D92 and above) and the "extremely high-surface-brightness arms" visible in optical images (Sandage \& Brucato 1979).

\subsubsection{NGC 1808}

The $1.34 \mathrm{GHz}$ continuum emission of NGC 1808 is almost unresolved by our observations. The resulting image does not add new information to previously published results (Dahlem et al. 1990; Saikia et al. 1990). The total flux derived from the present data is $616 \pm 15 \mathrm{mJy}$, which is about $18 \%$ higher than our earlier result of $523 \pm 10 \mathrm{mJy}$ at $1.49 \mathrm{GHz}$ (Dahlem et al. 1990). Partly, this might be explained by the different observing frequencies, but part of the discrepancy might be due to an intrinsic variability, possibly of the low-luminosity AGN (Véron-Cetty \& Veron 1985; Awaki \& Koyama 1993; Awaki et al. 1996). 


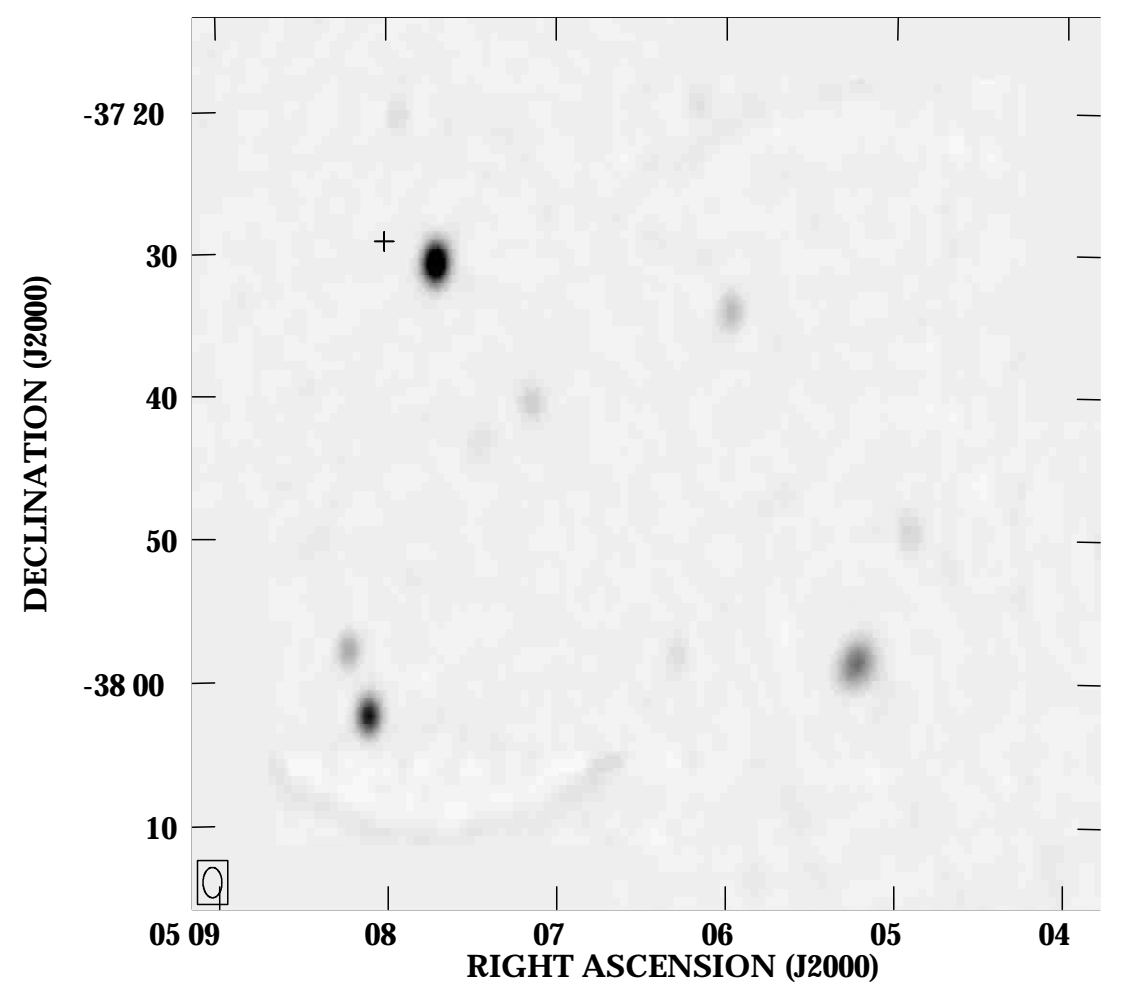

Fig. 5. Image of the $1.34 \mathrm{GHz}$ radio continuum emission of NGC 1808 (upper left) and NGC 1792 (lower right), the two dominant members of the NGC 1808 group of galaxies, and their surroundings. The cross in the northeastern spiral arm of NGC 1808 marks the position of SN1993af. The angular resolution of the data is indicated in the lower left; the linear grey scale ranges from 0 to the maximum observed surface brightness of $484 \mathrm{mJy}_{\text {beam }}{ }^{-1}$. The rms noise is about $1 \mathrm{mJy}^{\text {beam }}{ }^{-1}$ near the centre and $2 \mathrm{mJy}$ beam $^{-1}$ in the vicinity of SN1993af.

We checked our data for continuum emission from the type Ia supernova SN1993af at $\alpha, \delta(2000)=05^{\mathrm{h}} 08^{\mathrm{m}} 00.79,-37^{\circ} 29^{\prime} 12^{\prime \prime} .8$ (Hamuy \& Maza 1993; Biegelmayer et al. 1993), in the north-eastern outer spiral arm of NGC 1808. We have not detected any significant radio continuum emission at the position of the supernova, with a 1- $\sigma$ noise level of our map in this region of about $2.5 \mathrm{mJy}^{\text {beam }^{-1}}$.

\subsubsection{Comparing the radio continuum properties}

Considering that NGC 1808 is a classical starburst galaxy, while its companion NGC 1792 is a "normal" spiral, it is noteworthy that NGC 1792's total radio continuum flux density at $1.34 \mathrm{GHz}$ is more than half that of NGC 1808 (Table 3; see also Junkes et al. 1995). The major difference between the two galaxies does not lie in the total energy output, but in the size of the region over which the energy is produced in stellar winds and supernovae (Leitherer \& Heckman 1995) and released into the ISM.

In NGC 1808 , about $90 \%$ of the radio continuum emission arises from the central starburst, i.e. inside a region of $16^{\prime \prime} \times 10^{\prime \prime}$ (Saikia et al. 1990). Assuming an inclination angle of about $60^{\circ}$ and thus circular symmetry of the starburst, with a major axis extent of $16^{\prime \prime}$, this corresponds to an area of about $0.56 \mathrm{kpc}^{2}$. Based on a total radio contin- uum flux of 616 mJy at $1.34 \mathrm{GHz}$, this implies that about $555 \mathrm{mJy}$ arise from the central region.

At the distance of NGC 1808 of $10.9 \mathrm{Mpc}$, this corresponds to a radio luminosity of $L_{1.34}=8.75 \times$ $10^{20} \mathrm{~W} \mathrm{~Hz}^{-1}$. Using Eqs. (18), (20) and (21) by Condon (1992), and assuming that practically all radio continuum emission at $1.34 \mathrm{GHz}$ is synchrotron radiation, this radio luminosity can directly be translated into a supernova rate, $\nu_{\mathrm{SN}}$, and a star formation rate for stars with $M \geq 5 M_{\odot}, S F R$ (see Table 3 ). For NGC 1808 a mean radio spectral index of $\bar{\alpha}=-0.88\left(S \propto \nu^{\alpha}\right)$ was adopted (Dahlem et al. 1990). Strictly speaking, this value is valid only for the central starburst, but since the radio emission of the starburst dominates the total flux, it should be a good approximation of the overall spectral index. From this, it follows that the normalised SFR within the circumnuclear starburst in NGC 1808 (based on $90 \%$ of the total nonthermal radio continuum flux density) is approximately $34.1 M_{\odot} \mathrm{yr}^{-1} \mathrm{kpc}^{-2}$.

In NGC 1792 the SF activity is much more widespread, out to a radius of $2.2(7.0 \mathrm{kpc}$; D92), leading to an area over which SF is observed, again under the assumption of circular symmetry, of $153.1 \mathrm{kpc}^{2}$. Based on a radio spectral index of $\bar{\alpha}=-0.82$ (D92), the total $S F R$ following from the observed $1.34 \mathrm{GHz}$ flux density of $325 \mathrm{mJy}$ is $11.0 M_{\odot} \mathrm{yr}^{-1}$, which then translates into 
a normalised $S F R$ per unit area in NGC 1792 of about $0.072 M_{\odot} \mathrm{yr}^{-1} \mathrm{kpc}^{-2}$.

Thus, the primary reason for the different excitation conditions of the ISM in NGC 1792 and NGC 1808 and the prominent outflow from the central starburst region of NGC 1808, which has no equivalent in NGC 1792, is the difference in area over which the energy produced by stellar winds and supernovae is released, as reflected by the observed difference in radio continuum surface brightnesses in Fig. 5. This dilution effect alone leads to a difference in the average energy density by a factor of roughly 270 , while the total difference in the normalised $S F R$ between the disk of NGC 1792 and the central starburst in NGC 1808 is a factor of 474 .

For more detailed information on the SF activity in both galaxies see e.g. Saikia et al. (1990), Forbes et al. (1992), Phillips (1993), Collison et al. (1994), Dahlem et al. (1994), Krabbe et al. (1994), Mazzolini \& Webster (1996) and Kotilainen et al. (1996). The high-resolution rotation pattern of $\mathrm{H}$ I gas seen in absorption against the starburst in NGC 1808 is described by Koribalski et al. (1996). The distribution of stars in the central $2^{\prime} \times 2^{\prime}$ of both galaxies can be seen in the NIR $H$ band images by Jungwiert et al. (1997).

Acknowledgements. We would like to thank L. Staveley-Smith for his help in the calculation of the flux density to column density conversion and D. Malin for communicating the absence of intergalactic features on his unpublished deep optical images of the NGC 1808 group of galaxies. The Digitized Sky Surveys were produced at the Space Telescope Science Institute under U.S. Government grant NAG W-2166. The images of these surveys are based on photographic data obtained using the Oschin Schmidt Telescope on Palomar Mountain and the UK Schmidt Telescope. The plates were processed into the present compressed digital form with the permission of these institutions.

\section{References}

Awaki, H., \& Koyama, K. 1993, Adv. Space Res., 13, 221 Awaki, H., Ueno, S., Koyama, K., et al. 1996, PASJ, 48, 409 Bergh van den, S. 1978, Vistas in Astronomy, 22, 307

Biegelmayer, M., et al. 1993, IAU Circ., 5895

Blitz, L., Spergel, D. N., Teuben, P. J., Hartmann, D., \& Burton, W. B. 1999, ApJ, 514, 818

Bosma, A. 1981, AJ, 86, 1825

Brinks, E. 1990, in The Interstellar Medium in Galaxies, Proc. of the 2nd Teton Conf., Grand Teton National Park (Kluwer, Dordrecht)

Chromey, F. R., Elmegreen, D. M., Mandell, A., \& McDermott, J. 1998, AJ, 115, 2331
Collison, P. M., Saikia, D. J., Pedlar, A., et al. 1994, MNRAS, 268, 203

Combes, F. 1978, A\&A, 65, 47

Condon, J. J. 1992, ARAA, 30, 575

Dahlem, M. 1992, A\&A, 264, 483

Dahlem, M., Aalto, S., Klein, U., et al. 1990, A\&A, 240, 237

Dahlem, M., Bomans, D. J., \& Will, J.-M. 1994, ApJ, 432, 590

Dahlem, M., Ehle, M., \& Ryder, S. D. 2001, A\&A, 371, 45

Dahlem, M., Weaver, K. A., \& Heckman, T. M. 1998, ApJS, 118,401

Donahue, M., Aldering, G., \& Stocke, J. T. 1995, ApJ, 450, L45

Forbes, D. A., Boisson, C., \& Ward, M. J. 1992, MNRAS, 259, 293

Frater, R. H., Brooks, J. W., \& Whiteoak, J. B. 1992, J. Electr. Electron. Eng., 12, 103

Giovanelli, R., \& Haynes, M. P. 1988, in Galactic and Extragalactic Radio Astronomy, ed. G. L. Verschuur, \& K. I. Kellermann (Springer Verlag, Heidelberg), 524

Hamuy, M., \& Maza, J. 1993, IAU Circular, 5895

Haynes, M. P., Giovanelli, R., \& Roberts, M. S. 1979, ApJ, 229,83

Garcia, A. M. 1993, A\&AS, 100, 47

Jungwiert, B., Combes, F., \& Axon, D. J. 1997, A\&AS, 125, 479

Junkes, N., Zinnecker, H., Hensler, G., Dahlem, M., \& Pietsch, W. 1995, A\&A, 294, 8

Kenney, J. D., \& Young, J. S. 1989, ApJ, 344, 171

Koribalski, B., Dahlem, M., Mebold, U., \& Brinks, E. 1993, A\&A, 268, 14

Koribalski, B., Dettmar, R.-J., Mebold, U., \& Wielebinski, R. 1996, A\&A, 315, 71

Kotilainen, J. K., Forbes, D. A., Moorwood, A. F. M., van der Werf, P. P., \& Ward, M. J. 1996, A\&A, 313, 771

Krabbe, A., Sternberg, A., \& Genzel, R. 1994, ApJ, 425, 72

Leitherer, C., \& Heckman, T. M. 1995, ApJS, 96, 9

Mazzolini, M., \& Webster, R. 1996, PASA, 13, 107

Mirabel, F., et al. 1992, A\&A, 256, L22

Oort, J. H. 1966, Bull. Astr. Inst. Neth., 18, 421

Phillips, A. C. 1993, AJ, 105, 486

Roberts, M. S. 1975, in Galaxies and the Universe, ed. A. Sandage, M. Sandage, \& J. Kristian (Univ. of Chicago Press, Chicago)

Roberts, M. S., \& Haynes, M. P. 1994, ARA\&A, 32, 115

Saikia, D. J., Unger, S. W., Pedlar, A., et al. 1990, MNRAS, 245,397

Sandage, A., \& Brucato, R. 1979, AJ, 84, 472

Sérsic, J. L., \& Pastoriza, M. 1965, PASP, 77, 287

Shull, J. M., Roberts, D., Giroux, M. L., et al. 1999, AJ, 118, 1450

Véron-Cetty, M.-P., \& Véron, P. 1985, A\&A, 145, 425

Weliachew, L., Sancisi, R., \& Guélin, M. 1978, A\&A, 65, 37

Zwaan, M. A. 2001, [astro-ph/0103328] 